\title{
Ácidos graxos ômega 3 e tratamento da esquizofrenia
}

\section{Omega 3 fatty acid and schizophrenia treatment}

\author{
Juliane Costa Silva Zemdegs ${ }^{1}$, Gustavo Duarte Pimentel ${ }^{1}$, Margareth Rose Priel ${ }^{2}$
}

1 Programa de Pós-Graduação em Nutrição da Universidade Federal de São Paulo (Unifesp).

2 Pós-Graduação e Pesquisa do Centro Universitário São Camilo.

Recebido: 15/4/2009 - Aceito: 9/6/2009

\begin{abstract}
Resumo
Contexto: A esquizofrenia é uma desordem psiquiátrica complexa e debilitante cujo tratamento de base é realizado com medicamentos antipsicóticos. No entanto, evidências sugerem que a suplementação dietética com ácidos graxos ômega 3 (n-3) pode ser benéfica em diversas desordens psiquiátricas. Objetivo: Revisar a eficácia do n-3 como coadjuvante no tratamento farmacológico da esquizofrenia. Métodos: Realizou-se uma pesquisa nas bases de dados eletrônicas Medline, Lilacs e SciELO. A estratégia de busca também incluiu a busca em árvore. Todos os estudos randomizados e controlados relevantes foram incluídos nesta revisão, independentemente do ano de publicação. Resultados: Até o momento, foram divulgados seis estudos randomizados, duplo-cegos placebo controlados; cinco deles apresentaram resultados positivos na melhora dos sintomas da esquizofrenia, assinalando, ainda, superioridade do ácido graxo eicosapentaenoico (EPA) em relação ao ácido graxo docosaexaenoico. Em geral, o consumo de $2 \mathrm{~g} /$ dia de EPA conjuntamente com a medicação antipsicótica usual parece reduzir a sintomatologia da esquizofrenia, particularmente os sintomas positivos. Conclusão: A terapia nutricional com EPA mostrou-se útil como coadjuvante no tratamento da esquizofrenia. Por conseguinte, sugere-se que os pacientes esquizofrênicos sejam encorajados a consumir refeições balanceadas e saudáveis ricas em EPA e, caso a quantidade ideal não seja atingida pela dieta, a suplementação pode ser benéfica.
\end{abstract}

Zemdegs JCS, et al. / Rev Psiq Clín. 2010;37(5):223-7

Palavras-chave: Esquizofrenia, nutrição, ácido eicosapentaenoico, ácido graxo ômega 3, tratamento.

\begin{abstract}
Background: Schizophrenia is a complex and debilitating psychiatric disorder, whose primary pharmacological intervention is the use of antipsychotics. There is, however, growing evidence that dietary supplementation with omega 3 fatty acids (n-3) may be beneficial in several psychiatric conditions. Objective: To review the efficacy of n-3 as a treatment for schizophrenia. Methods: Electronic searches of the following databases were performed: Medline, Lilacs e SciELO. The search strategy also included cited reference searching. All relevant randomized controlled trials were included in the review. Results: To date, five out of six randomized, double-blind, and placebo controlled studies obtained improvement in the symptoms of the psychosis. Besides, an advantage in the intake of eicosapentaenoic fatty acid (EPA) in relation to docosahexaenoic fatty acid was designated. Essentially, the intake of $2 \mathrm{~g} /$ day of EPA in addition to the standard medication was effective in decreasing the symptoms of schizophrenia. Discussion: The nutritional therapy with EPA revealed to be useful as coadjutant in the treatment of schizophrenia. Therefore, we suggest that the schizophrenic patients should be encouraged to consume balanced and healthy meals rich in EPA and, if the ideal amount is not reached by the diet, the supplementation is likely to be beneficial.
\end{abstract}

Zemdegs JCS, et al. / Rev Psiq Clín. 2010;37(5):223-7

Keywords: Schizophrenia, nutrition, eicosapentaeinoic acid, omega 3 fatty acid, treatment.

\section{Introdução}

Depois do tecido adiposo, o sistema nervoso central possui o maior conteúdo de lipídios do corpo humano, e 35\% dos lipídios de um encéfalo adulto são ácidos graxos poli-insaturados ${ }^{1-3}$.

Os ácidos graxos poli-insaturados atuam na sinalização celular, regulação enzimática, síntese de eicosanoides, regulação da migração neuronal, determinação da plasticidade sináptica e modulação de citocinas que possuem atividade neuromodulatória e neurotransmissora ${ }^{4-10}$, de maneira que possivelmente estejam envolvidos na fisiopatologia de alguns transtornos psiquiátricos como a esquizofrenia e a depressão $0^{11-13}$.

Apesar da etiologia incerta da esquizofrenia, evidências apontam para um metabolismo anormal dos fosfolipídios, particularmente na composição dos ácidos graxos das membranas celulares ${ }^{14-19}$. Nesse contexto, estudos revelaram que pacientes esquizofrênicos apresentam níveis reduzidos de ácidos graxos de cadeia longa nas membranas dos eritrócitos, provavelmente por causa de atividade excessiva de uma fosfolipase $\mathrm{A}_{2}{ }^{20,21}$.

É possível que alterações dietéticas agravem ou aliviem os sintomas da esquizofrenia. Nesse sentido, Christensen e Christensen ${ }^{22}$ encontraram correlação positiva entre a melhora do prognóstico da esquizofrenia e o baixo consumo de gorduras totais e de gorduras de fontes animais - compostas principalmente por ácidos graxos saturados. Adicionalmente, observaram tendência de relação positiva entre o alto consumo de gorduras provenientes de vegetais, peixes e animais marinhos (ricos em ácidos graxos insaturados) e melhor desfecho da psicose em questão. Corroborando esse estudo, associação positiva entre o consumo de carne vermelha, laticínios e açúcar e pior prognóstico da esquizofrenia foi demonstrada e a baixa ingestão do ácido graxo ômega 3 (n-3) eicosapentaenoico (EPA) foi associada à presença de sintomas mais severos da esquizofrenia ${ }^{14}$.

Estudos preliminares relataram uma melhora dos sintomas da esquizofrenia após a suplementação de n-3 como terapia coadjuvante aos antipsicóticos ${ }^{23-26}$. Com base nessa perspectiva e tendo em vista que, apesar do avanço farmacológico ocorrido nas últimas décadas, o alcance da remissão completa desses pacientes ainda constitui um grande desafio ${ }^{27}$, o presente trabalho teve por objetivo revisar os estudos randomizados, duplo-cegos e placebo-controlados que avaliaram os efeitos da suplementação de n-3 como tratamento da esquizofrenia. 


\section{Métodos}

A busca bibliográfica consistiu na pesquisa de artigos publicados nas seguintes bases de dados eletrônicas: Medline (National Library of Medicine), Lilacs (Literatura Latino-americana e do Caribe em Ciências da Saúde) e SciELO (Scientific Electronic Library Online). A estratégia de busca foi a lógica booleana "e" para os seguintes descritores de saúde: dieta, nutrição, ácido graxo ômega 3 e o sistema nervoso central, combinados com o termo esquizofrenia. Idioma e período de publicação não foram restringidos. Todos os ensaios clínicos randomizados, duplo-cegos e placebo-controlados que utilizaram ácido graxo ômega 3 para o tratamento de pacientes esquizofrênicos foram incluídos na presente análise, independentemente do gênero, idade e raça dos participantes. Os estudos nos quais mais de $30 \%$ dos participantes não deram continuidade ao tratamento foram excluídos desta revisão. Para os artigos não disponíveis em território nacional, os respectivos autores foram contatados por e-mail a fim de se solicitar o envio dos artigos na íntegra.

\section{Resultados}

\section{Descrição dos estudos selecionados}

A busca eletrônica retornou 13 ensaios clínicos que avaliaram os efeitos da suplementação de n-3 sobre os sintomas de pacientes esquizofrênicos. Desses 13 artigos, 7 foram excluídos por não serem randomizados, duplo-cegos ou placebo-controlados.

Excluindo os pacientes que não seguiram o tratamento, os seis artigos incluídos nesta revisão randomizaram no total 381 pacientes. Em todos os estudos, o DSM-IV (Diagnostic and Statistical Manual of Mental Disorders) foi o critério para o diagnóstico da esquizofrenia e diversos escores foram utilizados para monitorar a gravidade da doença.
Fenton et al. ${ }^{28}$ também incluíram pacientes com desordens esquizoafetivas. Emsley et al. ${ }^{29}$ não referiram o gênero dos participantes, enquanto os outros estudos incluíram ambos os gêneros (feminino e masculino) nas análises. A idade dos pacientes analisados variou entre 15 e 65 anos, e o tamanho da amostra variou entre 30 e 122 participantes. Todos os estudos suplementaram n-3 e o compararam com a suplementação de placebo. O estudo de Peet et al. ${ }^{30}$ foi o único no qual os participantes interromperam a medicação antipsicótica durante a suplementação de n-3 ou placebo. Fenton et al. ${ }^{28}$ compararam diferentes doses de etil-EPA com o placebo.

\section{Suplementação de n-3 em pacientes esquizofrênicos: ensaios clínicos}

As características dos estudos randomizados, duplo-cegos e placebocontrolados realizados até o momento que avaliaram os efeitos da suplementação de n-3 em pacientes esquizofrênicos estão apresentadas na tabela 1, enquanto as características dos estudos excluídos estão na tabela 2.

Em 2001, Peet et al. ${ }^{30}$ realizaram um estudo clínico duplo-cego randomizado com 45 pacientes que receberam diariamente óleo rico em ácido graxo eicosapentaenoico (EPA) - 2 g/dia - ou óleo rico em ácido graxo docosaexaenoico (DHA) - $2 \mathrm{~g} / \mathrm{dia}$ - ou placebo por três meses. Os autores relataram melhora no escore PANSS (Positive and Negative Syndrome Scale) no grupo EPA, enquanto a resposta terapêutica no grupo tratado com DHA não diferiu do placebo. O efeito do EPA sobre os sintomas positivos da doença foi superior ao DHA tanto pela análise de variância para medidas repetidas como pela porcentagem de melhora dos sintomas.

Peet e Horrobin ${ }^{31}$ averiguaram os efeitos da suplementação de diferentes doses de etil-EPA (1, 2 ou $4 \mathrm{~g} / \mathrm{dia})$ ou placebo por 12 semanas em 115 pacientes esquizofrênicos sob medicação antipsi-

Tabela 1. Características dos estudos randomizados, duplo-cegos que avaliaram os efeitos da suplementação de n-3 no tratamento de pacientes esquizofrênicos

\begin{tabular}{|c|c|c|c|}
\hline Estudo & Participantes & Intervenção & Resultados \\
\hline Peet, 2001a30 & $\begin{array}{l}\text { Dg: Esquizofrenia; } N \text { = } 55 \\
\text { Gênero: } 30 \text { đe e } 15 \text { +; Idade: } 30-56 \text { anos; } \\
\text { Perdas: } 10\end{array}$ & $\begin{array}{l}\text { Óleo enriquecido com EPA (2 g/dia de EPA) } \\
\text { Óleo enriquecido com DHA } \\
\text { Placebo (óleo de milho) } \\
\text { Duração: } 12 \text { semanas }\end{array}$ & $\begin{array}{l}\text { EPA }>\text { DHA = placebo para o estado mental } \\
\text { (PANSS) }\end{array}$ \\
\hline Peet, 2001b30 & $\begin{array}{l}\text { Dg: Esquizofrenia; } N \text { = 30; Gênero: } 18 \text { ô e } \\
12 \text { + ; Idade: } 25-45 \text { anos; Perdas: } 4\end{array}$ & $\begin{array}{l}\text { Monoterapia com óleo enriquecido com EPA } \\
\text { (2 g/dia de EPA); Óleo enriquecido com DHA; } \\
\text { Placebo (óleo de milho); Duração: } 12 \text { semanas }\end{array}$ & $\begin{array}{l}\text { EPA > placebo para o estado mental (PANSS). } \\
\text { Todos os pacientes do grupo placebo }(n=12) \\
\text { e } 8 \text { do grupo EPA }(n=14) \text { necessitaram de } \\
\text { antipsicótico e o grupo mantido somente com } \\
\text { EPA apresentou melhora na PANSS }\end{array}$ \\
\hline Fenton, 200128 & 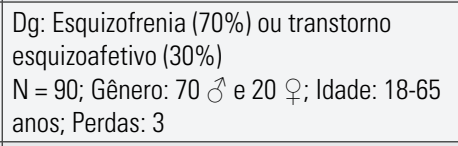 & $\begin{array}{l}3 \mathrm{~g} / \text { dia de etil-EPA; Placebo; Duração: } \\
16 \text { semanas }\end{array}$ & $\begin{array}{l}\text { EPA = placebo para o estado mental (PANSS, } \\
\text { MADRS), estado global (CGI-S), efeitos } \\
\text { adversos (AIMS, SAS) }\end{array}$ \\
\hline Emsley, 200229 & $\begin{array}{l}\text { Dg: Esquizofrenia; N = 40; Gênero: não } \\
\text { mencionado; Idade: 18-55 anos; Perdas: } 1\end{array}$ & $\begin{array}{l}3 \mathrm{~g} / \text { dia de etil-EPA; Placebo (parafina líquida); } \\
\text { Duração: } 12 \text { semanas }\end{array}$ & EPA $>$ placebo na escala PANSS e EPRS \\
\hline Peet e Horrobin, $2002^{31}$ & $\begin{array}{l}\text { Dg: Esquizofrenia; } N=122 ; \text { Gênero: } 66 \text { ô e } \\
39 \text { क; Idade: 25-45 anos; Perdas: } 7\end{array}$ & $\begin{array}{l}\text { Etil-EPA: } 1 \text { g/dia; } 2 \text { g/dia; } 4 \text { g/dia; Placebo } \\
\text { Duração: } 12 \text { semanas }\end{array}$ & $\begin{array}{l}\text { EPA = placebo (análise primária) para o } \\
\text { estado mental (PANSS, MADRS) } \\
\text { EPA }(2 \mathrm{~g})>\text { placebo no subgrupo clozapina } \\
\text { para o estado mental (PANSS, MADRS) } \\
\text { Efeitos adversos AINS, SAS }\end{array}$ \\
\hline Berger et al., 200732 & $\begin{array}{l}\text { Dg: } 1^{0} \text { episódio de psicose; N = 80; Gênero: } \\
53 \text { ô e } 16 \text { q; Idade: } 15-29 \text { anos; Perdas: } 11\end{array}$ & $\begin{array}{l}2 \mathrm{~g} / \text { dia de etil-EPA vs. placebo } \\
\text { Duração: } 12 \text { semanas }\end{array}$ & $\begin{array}{l}\text { EPA > placebo para escalas CDSS, CGI-S, } \\
\text { GAF, SANS, SAS, SOFAS, BPRS e o grupo } \\
\text { EPA reduziu em } 20 \% \text { o uso de antipsicóticos, } \\
\text { apresentou menos efeitos colaterais } \\
\text { extrapiramidais, menos constipação e menos } \\
\text { efeitos colaterais sexuais }\end{array}$ \\
\hline
\end{tabular}

Brief Psychiatric Rating Scale (BPRS); Positive and Negative Syndrome Scale (PANSS); Abnormal Involuntary Movement Scale (AIMS); Henrich's Quality of Life (00L) Scale; Montgomery-Asberg Depression Rating Scale (MADRS); Calgary Depression Scale for Schizophrenia (CDSS); Clinical Global Impressions-Severity of IIIness Scale (CGI-S); Extrapyramidal Symptom Rating Scale (EPRS); Global Assessment of Functioning scale (GAF); Scale for the Assessment of Negative Symptoms (SANS); Simpson-Angus Scale (SAS); Social and Occupational Functioning Assessment Scale (SOFAS); Eicosapentaenoic acid (EPA); Docosahesaenoic acid (DHA), Diagnóstico (Dg); Unidade Internacional (UI). 
Tabela 2. Estudos que avaliaram os efeitos da suplementação de ácidos graxos ômega 3 no tratamento da esquizofrenia e que foram excluídos da revisão da literatura

\begin{tabular}{|c|c|}
\hline Referência & Características e resultados do estudo \\
\hline Rudin, $1981^{33}$ & $\begin{array}{l}\text { Relato de cinco casos de pacientes esquizofrênicos } \\
\text { crônicos que receberam óleo de semente de linho } \\
\text { ( } 50 \% \text { ácido graxo alfa-linolênico) a uma dose de } \\
0,2-1,0 \mathrm{~g} / \mathrm{kg} / \text { dia. Melhoras nos sintomas da doença } \\
\text { foram observadas pelos pesquisadores e relatadas } \\
\text { pelos pacientes }\end{array}$ \\
\hline 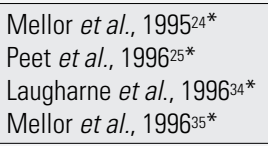 & $\begin{array}{l}\text { Vinte pacientes esquizofrênicos receberam } \\
\text { diariamente } 10 \mathrm{~g} \text { de óleo de peixe por seis semanas } \\
\text { e apresentaram melhora significativa nas escalas } \\
\text { PANSS }(p<0,002) \text { e AIMS }(p<0,002)\end{array}$ \\
\hline Puri et al., 200026 & $\begin{array}{l}\text { Relato de caso de um paciente esquizofrênico, } 31 \\
\text { anos de idade, sem medicação prévia. } 0 \text { tratamento } \\
\text { com emulsão rica em EPA ( } 30 \mathrm{~mL} / \text { dia) por seis meses } \\
\text { melhorou os sintomas positivos e negativos da } \\
\text { doença }\end{array}$ \\
\hline Su et al., 200136 & $\begin{array}{l}\text { Relato de caso de uma paciente esquizofrênica que } \\
\text { apresentou um episódio agudo de exacerbação da } \\
\text { psicose durante a gravidez. A monoterapia com } \\
\text { n-3 (4 g EPA/ } 2 \text { g DHA) por seis meses melhorou } \\
\text { os sintomas positivos e negativos da doença e não } \\
\text { apresentou efeitos colaterais }\end{array}$ \\
\hline
\end{tabular}

$\overline{\text { Ômega } 3 \text { (n-3); Ácido eicosapentaenoico (EPA); Ácido docosaexaenoico (DHA); Positive and }}$ Negative Syndrome Scale (PANSS); Abnormal Involuntary Movement Scale (AIMS); Brief Psychiatric Rating Scale (BPRS); Henrich's Quality of Life (OOL) Scale; Unidade Internacional (UI); * Apresentam as mesmas características e resultados.

cótica. Os pacientes que receberam $2 \mathrm{~g} /$ dia de etil-EPA associado ao tratamento com clozapina apresentaram melhora de $25 \%$ na escala PANSS. No entanto, um efeito placebo foi observado em pacientes sob medicação antipsicótica típica e atípica.

Fenton et al. ${ }^{28}$ randomizaram 87 pacientes portadores de esquizofrenia ou desordens esquizoafetivas sob medicação antipsicótica para receber $3 \mathrm{~g} /$ dia de etil-EPA ou placebo por 16 semanas. Segundo os autores, os grupos eram homogêneos, inclusive para o consumo diário de n-3. Não foram observadas diferenças significativas nos escores da PANSS em nenhum dos momentos analisados $(1,2,4,8$, 12 ou 16 semanas) e dois efeitos adversos foram relatados no grupo EPA: infecção do trato respiratório superior e diarreia. Uma análise secundária dos dados revelou que os pacientes tratados com clozapina e etil-EPA apresentaram melhoras significativas dos sintomas.

Emsley et al. ${ }^{29}$ randomizaram 40 pacientes esquizofrênicos para receber $3 \mathrm{~g} /$ dia de etil-EPA ou placebo durante três meses. O grupo EPA apresentou melhora significativa dos sintomas da doença quando comparado ao grupo placebo após 3, 9 e 12 semanas de tratamento. Segundo os autores, a ingestão alimentar de n-3 antes e durante o tratamento não diferiu entre os grupos. Ao final de 12 semanas de tratamento, o grupo etil-EPA apresentou melhora na escala PANSS e redução significativa nos escores da Extrapyramidal Symptom Rating Scale (ESRS).

A eficácia da suplementação de $2 \mathrm{~g} /$ dia de EPA como único tratamento para a esquizofrenia também foi investigada em 26 pacientes por Peet et al. ${ }^{30}$. Ao final de três meses de estudo clínico duplo-cego, todos os pacientes do grupo placebo requereram medicação antipsicótica, enquanto o grupo EPA apresentou melhora significativa na escala PANSS, particularmente para os sintomas positivos da doença, e metade desses pacientes não necessitou de tratamento convencional.

Segundo Berger et al. ${ }^{32}$, a cronicidade da doença e o uso estabelecido de medicação antipsicótica podem acentuar ou mascarar os efeitos do n-3. Por essa razão, esses autores avaliaram a eficácia da suplementação de $2 \mathrm{~g} /$ dia de etil-EPA por 12 semanas em pacientes com primeiro episódio de psicose recente que não tinham recebido medicação antipsicótica previamente ou que tinham sido recentemente tratados com antipsicóticos atípicos. Os pacientes foram submetidos a uma série de escalas de avaliação clínica (Tabela 1) após 3, 6, 9 e 12 semanas de tratamento. Os resultados sugerem que o etil-EPA pode acelerar a resposta ao tratamento e melhorar a tolerância às medicações antipsicóticas.

\section{Discussão}

Os estudos realizados até o momento são de grande interesse para a prática clínica. No entanto, algumas falhas em suas metodologias podem ser citadas: 1) variáveis que não foram controladas; 2) biodisponibilidade do etil-EPA; 3 ) as alterações observadas nas escalas de sintomas foram comparadas em relação ao escore basal, isto é, ao escore anterior à suplementação, e fornecidas em porcentagem de alteração; 4) número pequeno de participantes avaliados.

No que tange às variáveis não controladas, Horrobin ${ }^{37}$ acredita que as informações dos procedimentos experimentais no termo de consentimento podem ter induzido os pacientes a consumirem alimentos ricos em n-3 ou suplementos de óleo de peixe. Apesar da especulação, isso não foi averiguado.

Adicionalmente, o fato de alguns estudos não terem realizado uma avaliação da ingestão alimentar dos pacientes constitui uma variável de contradição às pesquisas e alude à necessidade de distinção entre necessidade dietética, suplementação e dose farmacológica de n-3 para pacientes esquizofrênicos.

As recomendações de n-3 para a população saudável estão apresentadas na tabela 3 , a partir da qual é possível observar que as doses que apresentaram benefícios no tratamento de pacientes esquizofrênicos são cerca de quatro vezes maiores do que as quantidades recomendadas para adultos saudáveis. No entanto, em decorrência dos dados de declínio da ingestão de n-3 pela humanidade ao longo dos séculos ${ }^{39,40}$, presume-se que seja possível que os pacientes esquizofrênicos obtenham a quantidade ótima de n-3 por meio da alimentação, não implicando, a princípio, necessidade de suplementação.

Quanto à biodisponibilidade do n-3, é importante lembrar que esse ácido graxo não é um composto isolado no seu estado natural, mas parte de moléculas naturalmente maiores, como os triacilgliceróis e os fosfolipídios. Para a obtenção de cápsulas de EPA ou DHA, essas moléculas precisam ser hidrolisadas, purificadas e estabilizadas a etil-éster. Esse procedimento resulta em um produto que não é mais natural, e sim químico, cuja biodisponibilidade é menor do que quando o n-3 é fornecido na forma de fosfolipídios ${ }^{41}$.

Ademais, o conceito de resposta pode apresentar alguns problemas quanto à sua interpretação, pois a utilização do critério de porcentagem de redução em relação ao escore basal não fornece uma informação precisa sobre como o paciente está no final do tratamento em relação à sintomatologia 42 .

Apesar das falhas e limitações discutidas, é surpreendente encontrar o nível de significância estatística que emergiu de forma considerável em um grupo pequeno de participantes. Além disso, o critério diagnóstico adotado nesses ensaios experimentais para inclusão dos pacientes foi o DSM-IV, que tende a selecionar pacientes com prognóstico menos favorável do que critérios mais inclusivos ${ }^{43}$.

Ainda que a maioria dos estudos avaliados na presente revisão não tenha relatado efeitos adversos associados à suplementação de n-3, os profissionais da área da saúde devem estar cientes de um possível aumento do tempo de sangramento, do peso corporal e dos níveis de colesterol total e HDL-colesterol ${ }^{44}$.

\section{Conclusão}

A compilação dos estudos clínicos experimentais evidenciou a relação entre a suplementação de EPA e a melhora dos sintomas da esquizofrenia. Por conseguinte, sugere-se que os pacientes esquizofrênicos sejam encorajados a consumirem refeições balanceadas e saudáveis ricas em EPA e, caso a quantidade ideal não seja atingida pela dieta, é possível que a suplementação de n-3 seja benéfica. Vale lembrar que, assim como em qualquer forma de tratamento, a terapia nutricional deve ser supervisionada e as doses ajustadas sempre que necessário para que os benefícios do tratamento sejam atingidos. 
Tabela 3. Recomendações de ingestão de n-3, EPA + DHA, segundo diferentes organizações governamentais e de saúde

\begin{tabular}{l|c|c}
\hline \multicolumn{1}{c|}{ Referências } & Ano & Recomendações \\
\hline Eurodiet Conference & 2000 & $200 \mathrm{mg} / \mathrm{dia}$ \\
\hline $\begin{array}{l}\text { Agence Française de Sécurité Sanitaire } \\
\text { des Aliments, Centre national de } \\
\text { Coordination des Études et Recherches } \\
\text { sur la Nutrition et L'Alimentation; Centre } \\
\begin{array}{l}\text { National de la Recherche Scientifique } \\
\text { (France) }\end{array}\end{array}$ & 2001 & $500 \mathrm{mg} / \mathrm{dia}$ \\
\hline $\begin{array}{l}\text { UK Scientific Adivisory Committee on } \\
\text { Nutrition }\end{array}$ & 2004 & $450 \mathrm{mg} / \mathrm{dia}$ \\
\hline $\begin{array}{l}\text { International Society for the Study of Fatty } \\
\text { acids and Lipids }\end{array}$ & 2004 & $500 \mathrm{mg} / \mathrm{dia}$ \\
\hline Health Council of the Netherlands & 2001 & $\mathrm{n}-3$ (PUFA): $450 \mathrm{mg} / \mathrm{dia}$ \\
\hline Superior Health Council of Belgium & 2002 & $0,3 \%$ ED (667 mg/dia) \\
\hline American Dietetic Association & 2007 & $500 \mathrm{mg} / \mathrm{dia}$ \\
\hline
\end{tabular}

Modificado de Harris et al.38. Ômega 3 (n-3); Ácido-insaturado eicosapentaenoico (EPA); Ácido docosaexaenoico (DHA); Energia diária (ED); Ácido graxo poli-ionsaturado (PUFA).

\section{Agradecimentos}

Ao Conselho Nacional de Pesquisa e Desenvolvimento Científico (CNPq), pelas bolsas de doutorado e mestrado cedidas, respectivamente, a Juliane Costa Silva Zemdegs e Gustavo Duarte Pimentel.

Não há conflitos de interesse.

\section{Referências}

1. Haag M. Essential fatty acids and the brain. Can J Psychiatry. 2003;48(3):195-203.

2. Berger GE, Wood SJ, Pantelis C, Velakoulis D, Wellard RM, McGorry PD. Implications of lipid biology for the pathogenesis of schizophrenia. Aust N Z J Psychiatry. 2002;36(3):355-66.

3. Bourre JM. Roles of unsaturated fatty acids (especially omega-3 fatty acids) in the brain at various ages and during ageing. J Nutr Health Aging. 2004;8(3):163-74.

4. Horrobin DF. The membrane phospholipid hypothesis as a biochemical basis for the neurodevelopmental concept of schizophrenia. Schizophr Res. 1998;30(3):193-208.

5. Bennett CN, Horrobin DF. Gene targets related to phospholipid and fatty acid metabolism in schizophrenia and other psychiatric disorders: an update. Prostaglandins Leukot Essent Fatty Acids. 2000;63(1-2):47-59.

6. Peet M. Essential fatty acids: theoretical aspects and treatment implications for schizophrenia and depression. Advances in Psychiatric Treatment. 2002;8:223-9.

7. Fenton WS, Hibbeln J, Knable M. Essential fatty acids, lipid membrane abnormalities, and the diagnosis and treatment of schizophrenia. Biol Psychiatry. 2000;47(1):8-21.

8. Carrié I, Clément M, de Javel D, Francès H, Bourre JM. Specific phospholipid fatty acid composition of brain regions in mice. Effects of $n-3$ polyunsaturated fatty acid deficiency and phospholipid supplementation. J Lipid Res. 2000;41(3):465-72.

9. Peet M. Nutrition and schizophrenia. World Rev Nutr Diet. 2005;95:17-28.

10. Burdge GC, Jones AE, Wootton SA. Eicosapentaenoic and docosapentaenoic acids are the principal products of alpha-linolenic acid metabolism in young men. Br J Nutr. 2002;88(4):355-63.

11. Peet M, Stokes C. Omega-3 fatty acids in the treatment of psychiatric disorders. Drugs. 2005; 65(8):1051-9.

12. Bourre JM. Where to find omega- 3 fatty acids and how feeding animals with diet enriched in omega- 3 fatty acids to increase nutritional value of derived products for human: what is actually useful ? J Nutr Health Aging. 2005;9(4):232-42.

13. Lakhan SE, Vieira KF. Nutritional therapies for mental disorders. Nutr J. 2008;7:2.

14. Laugharne JD, Mellor JE, Peet M. Fatty acids and schizophrenia. Lipids. 1996;31(Suppl):S163-5.
15. Peet M. Eicosapentaenoic acid in the treatment of schizophrenia and depression: rationale and preliminary double-blind clinical trial results. Prostaglandins Leukot Essent Fatty Acids. 2003;69(6):477-85.

16. Reddy RD, Yao JK. Environmental factors and membrane polyunsaturated fatty acids in schizophrenia. Prostaglandins Leukot Essent Fatty Acids. 2003;69(6):385-91.

17. Gattaz WF. Neuroquímica da esquizofrenia: papel dos fosfolípides. Rev Bras Psiquiatr. 2000;22(Supl 1):5-8.

18. Tsaluchidu S, Cocchi M, Tonello L, Puri BK. Fatty acids and oxidative stress in psychiatric disorders. BMC Psychiatry. 2008;8(Suppl 1):S5.

19. Emsley R, Oosthuizen P, Van Rensburg SJ. Clinical potential of omega-3 fatty acids in the treatment of schizophrenia. CNS Drugs. 2003;17(15):1081-91.

20. Gattaz WF. Phospholipase A2 in schizophrenia. Biol Psychiatry. 1992;31:214-6.

21. Gattaz WF, Schmitt A, Maras A. Increased platelet phospholipase A2 activity in schizophrenia. Schizophr Res. 1995;16:1-6.

22. Christensen O, Christensen E. Fat consumption and schizophrenia. Acta Psychiatr Scand. 1988;78(5):587-91.

23. Su KP, Shen WW, Huang SY. Omega-3 fatty acids as a psychotherapeutic agent for a pregnant schizophrenic patient. Eur Neuropsychopharmacol. 2001;11(4):295-9.

24. Mellor JE, Laugharne JDE, Peet M. Schizophrenic symptoms and dietary intake of n-3 fatty acids. Schizophr Res. 1995;18:85-6.

25. Peet M, Laugharne JDE, Mellor J, Ramchand CN. Essential fatty acid deficiency in erythrocyte membranes from chronic schizophrenic patients, and the clinical effects of dietary supplementation. Prostaglandins Leukot Essent Fatty Acids. 1996;55:71-5.

26. Puri BK, Richardson AJ, Horrobin DF, Easton T, Saeed N, Oatridge A et al. Eicosapentaenoic acid treatment in schizophrenia associated with symptom remission, normalization of blood fatty acids, reduced neuronal membrane phospholipid turnover and structural brain changes. Int $J$ Clin Pract. 2000;54(1):57-63.

27. Barreto EMP, Elkis H. Evidências de eficácia da terapia cognitiva comportamental na esquizofrenia. Rev Psiq Clín. 2007;34(Supl 2):204-7.

28. Fenton WS, Dickerson F, Boronow J, Hibbeln JR, Knable M. A placebocontrolled trial of omega-3 fatty acid (ethyl eicosapentaenoic acid) supplementation for residual symptoms and cognitive impairment in schizophrenia. Am J Psychiatry. 2001;158(12):2071-4.

29. Emsley R, Myburgh C, Oosthuizen P, Van Rensburg SJ. Randomized, placebo-controlled study of ethyl-eicosapentaenoic acid as supplemental treatment in schizophrenia. Am J Psychiatry. 2002;159(9):1596-8.

30. Peet M, Brind J, Ramchand CN, Shah S, Vankar GK. Two double-blind placebo-controlled pilot studies of eicosapentaenoic acid in the treatment of schizophrenia. Schizophr Res. 2001;49(3):243-51.

31. Peet M, Horrobin DF. E-E Multicentre Study Group. A dose-ranging exploratory study of the effects of ethyl-eicosapentaenoate in patients with persistent schizophrenic symptoms. J Psychiatr Res. 2002;36(1):7-18.

32. Berger GE, Proffitt, TM, McConchie M, Yuen H, Wood SJ, Amminger GP, et al. Ethyl-eicosapentaenoic acid in first-episode psychosis: a randomized, placebo-controlled trial. J Clin Psychiatry. 2007;68:1-9.

33. Rudin DO. The major psychoses and neuroses as omega- 3 essential fatty acid deficiency syndrome: substrate pellagra. Biol Psychiatry. 1981;16(9):837-50.

34. Laugharne JDE, Mellor JE, Peet M. Fatty acids and schizophrenia. Lipids. 1996;31(Suppl):163-5.

35. Mellor JE, Laugharne JDE, Peet M. Omega-3 fatty acid supplementation in schizophrenic patients. Hum Psychopharmacol. 1996;11:39-46.

36. Su KP, Shen WW, Huang SY. Omega-3 fatty acids as a psychotherapeutic agent for a pregnant schizophrenic patient. Eur Neuropsychopharmacol. 2001;11(4):295-9.

37. Horrobin DF. Omega-3 Fatty acid for schizophrenia. Am J Psychiatry. 2003;160(1):188-9.

38. Harris WS, Mozaffarian D, Lefevre M, Toner CD, Colombo J, Cunnane SC, et al. Towards establishing dietary reference intakes for eicosapentaenoic and docosahexaenoic acids. J Nutr. 2009;139:804S-19S.

39. Simopoulos AP, Leaf A, Salem N Jr. Workshop on the essentiality of and recommended dietary intakes for omega- 6 and omega- 3 fatty acids. J Am Coll Nutr. 1999;18(5):487-9.

40. Simopoulos AP. Human requirement for $\mathrm{N}-3$ polyunsaturated fatty acids Poult Sci. 2000;79(7):961-70. 
41. Wijendran V, Huang MC, Diau GY, Boehm G, Nathanielsz PW, Brenna JT. Efficacy of dietary arachidonic acid provided as triglyceride or phospholipid as substrates for brain arachidonic acid accretion in baboon neonates. Pediatr Res. 2002;51(3):265-72.

42. Elkis H. A remissão em esquizofrenia é possível? Rev Psiq Clín. 2007;34(Supl 2):160-3.
43. Menezes PR. Prognóstico da esquizofrenia. Rev Bras Psiquiatr. 2000;22(Supl 1):18-20.

44. Emsley R, Niehaus DJ, Oosthuizen PP, Koen L, Ascott-Evans B, Chiliza $B$, et al. Safety of the omega-3 fatty acid, eicosapentaenoic acid (EPA) in psychiatric patients: results from a randomized, placebo-controlled trial. Psychiatry Res. 2008;161(3):284-91. 